\title{
RESSECÇÃO CIRÚRGICA DE EXTENSO ADENOMA PLEOMÓRFICO EM GLÂNDULA SALIVAR ACESSÓRIA NA ÁREA DO PALATO DURO
}

\author{
SURGICAL REMOVAL OF AN EXTENSIVE PLEOMORPHIC ADENOMA IN ACCESSORY \\ SALIVARY GLAND IN THE HARD PALATE REGION
}

\author{
Caroline Hoffmann BUENO'1; Juliana Silveira EMERIM²; Alexandre Silva de \\ QUEVEDO $^{3}$; Deise PONZONI ${ }^{4}$;Adriana CORSETTI ${ }^{5}$; Angelo Luiz FREDDO ${ }^{6}$
}

${ }^{1}$ Mestre em Prótese Dentária; Residente do Programa de Residência Integrada em Saúde Bucal/ Cirurgia e Traumatologia Buco-maxilo-faciais da Faculdade de Odontologia da Universidade Federal do Rio Grande do Sul (UFRGS).

${ }^{2}$ Especialista em Saúde da Família e Comunidade; Especialista em Cirurgia e Traumatologia Buco-maxilofaciais.

${ }^{3}$ Doutor em Anatomia e Neurobiologia; Professor Adjunto do Departamento de Cirurgia e Ortopedia da Faculdade de Odontologia da UFRGS.

${ }^{4}$ Mestre e Doutora em Cirurgia e Traumatologia Bucomaxilo-faciais; Professora Titular do Departamento de Cirurgia e Ortopedia da Faculdade de Odontologia da UFRGS; Chefe da Unidade de Cirurgia Buco-maxilofacial do Hospital de Clínicas de Porto Alegre (HCPA).

${ }^{5}$ Mestre e Doutora em Cirurgia e Traumatologia Bucomaxilo-faciais; Professora Adjunta do Departamento de Cirurgia e Ortopedia da Faculdade de Odontologia da UFRGS.

${ }^{6}$ Mestre e Doutor em Cirurgia e Traumatologia Bucomaxilo-faciais; Professor Adjunto do Departamento de Cirurgia e Ortopedia da Faculdade de Odontologia da UFRGS.

Correspondência:

Nome: Caroline Hoffmann Bueno

Email: carolhbueno@gmail.com

Endereço: Travessa Miranda e Castro, 21, apto 312 90040-280, Porto Alegre/RS

\section{Resumo}

Introdução: O adenoma pleomórfico, uma neoplasia benigna, é uma das patologias mais comuns de glândula salivar, cujo tratamento é a excisão cirúrgica. O objetivo deste estudo é relatar um caso de adenoma pleomórfico de grande proporção em glândula salivar acessória na região do palato duro. Relato de caso: Paciente do sexo feminino, 22 anos, encaminhada de sua unidade básica de saúde devido a uma lesão em região de palato, assintomática, com evolução de 1 ano. No exame clínico intra-bucal observou-se um nódulo extenso em palato do lado direito. A biópsia incisional revelou o diagnóstico histopatológico de adenoma pleomórfico. A paciente foi submetida à excisão cirúrgica da lesão sob anestesia geral. Apresentou boa evolução pósoperatória, sem recidivas. Discussão: $O$ adenoma pleomórfico é uma neoplasia de diagnóstico clínico, com confirmação a partir de exame anatomopatológico. Embora seja uma patologia de crescimento lento, quando o tratamento não é realizado de maneira precoce, a lesão pode atingir grandes dimensões, aumentando a morbidade do procedimento cirúrgico e podendo levar a maiores taxas de recidiva e/ou transformação maligna. Conclusão: Salientamos a importância de um diagnóstico preciso e um tratamento efetivo com a maior brevidade. Quando possível, as próteses obturadoras/aparelhos intraorais acrílicos podem e devem ser utilizados de forma associada aos tratamentos cirúrgicos de tumores; possibilitando o reestabelecimento da função e do conforto pós-operatório do paciente.

Palavras-chave: Adenoma pleomorfo. Neoplasias das glândulas salivares. Palato duro. Cirurgia bucal. 


\begin{abstract}
Introduction: The pleomorphic adenoma, a benign neoplasm, is one of the most common pathologies of the salivary gland. The treatment of choice for pleomorphic adenoma is surgical excision. The aim of this study is to report a case of a large pleomorphic adenoma located in accessory salivary gland in the hard palate region. Case report: A 22-year-old female patient was referred from her primary health unit due to an asymptomatic lesion located in the hard palate with a 1-year evolution. The intra-oral examination revealed an extensive nodule on the right side of the palate. The examination of the incisional biopsy revealed the histopathological diagnosis of pleomorphic adenoma. The patient underwent surgical excision, under general anesthesia, presenting a good postoperative evolution, without signs of recurrence. Discussion: The diagnosis of pleomorphic adenoma is a combination of the clinical aspects with the anatomopathological examination. Although it is a slow growing lesion, when treatment is not early performed, it may reach large dimensions, leading to an increased morbidity of the surgical procedure and to higher rates of recurrence and/or malignant transformation. Conclusion: We emphasize the importance of accurate diagnosis and effective treatment at the earliest possible time. When possible, acrylic intraoral prostheses/obturators may be used in association with surgical approaches, restoring function and increasing the comfort of the postoperative period.
\end{abstract}

Keywords: Pleomorphic adenoma. Salivary gland neoplasms. Hard palate. Oral surgery.

\title{
Introdução
}

Dentre as patologias tratadas pela cirurgia buco-maxilo-facial, os tumores de glândulas salivares (TGS) apesar de infrequentes, não são incomuns ${ }^{1}$. A incidência anual mundial dos TGS varia de cerca de 1,0 a 6,5 casos por 100.000 indivíduos. Diante disso, salienta-se a importância do diagnóstico clínico adequado e sua diferenciação com outras patologias, como por exemplo, processos inflamatórios ${ }^{1,2}$.

O adenoma pleomórfico (AP), patologia mais comum de glândula salivar, é uma neoplasia benigna cujas principais características clínicas são: aumento de volume de consistência firme, superfície lisa, indolor e com tempo de evolução lento, ocorrendo mais frequentemente em adultos jovens e adultos de meia idade, com discreta preferência pelo sexo feminino ${ }^{2}$. O AP representa cerca de $53 \%$ a $77 \%$ dos tumores de parótida, $44 \%$ a $68 \%$ dos tumores da glândula submandibular e $33 \%$ a $43 \%$ dos tumores de glândula salivar menor ou acessória ${ }^{2-6}$. Na região intra-bucal, a localização mais comum do AP é o palato, especialmente em região lateral e posterior. Histopatologicamente, normalmente apresenta-se como uma lesão encapsulada e bem circunscrita, composta de epitélio glandular, células mioepiteliais e fundo similar ao mesênquima ${ }^{2,3,4,5,6}$.

O tratamento do AP é cirúrgico. Somente a excisão cirúrgica completa garante a remoção total da cápsula e das células tumorais. Quando o AP acomete o palato duro, a excisão deve ocorrer abaixo do periósteo, incluindo a mucosa sobreadjacente $e^{2,3,4,5,6}$. O tratamento adequado leva a uma taxa de cura de mais de $95 \%$, porém, devemos citar que este tumor tem potencial à transformação maligna em 5\% dos casos para carcinoma exadenoma pleomórfico e, portanto, não deve ser negligenciado ${ }^{2,3,4,5,6}$.

O objetivo deste trabalho é relatar um caso de adenoma pleomórfico de grande proporção em glândula salivar acessória de palato duro, diagnosticado no Hospital de 
Clínicas de Porto Alegre (HCPA), onde realizou-se a excisão cirúrgica completa e colocação de um aparelho acrílico para proteção da ferida cirúrgica.

\section{Relato de caso}

Paciente VSE, 22 anos, sexo feminino, residente de Porto Alegre, Rio Grande do Sul, Brasil, compareceu ao ambulatório de Cirurgia Buco-Maxilo-Facial do HCPA, encaminhada de sua unidade básica de saúde devido a uma lesão em região de palato, assintomática, com evolução aproximadamente de 1 ano, de acordo com a paciente. Em sua história médica prévia, paciente não referiu utilização de nenhuma medicação de uso contínuo, negou alcoolismo e tabagismo. No exame clínico intra-bucal, observou-se um

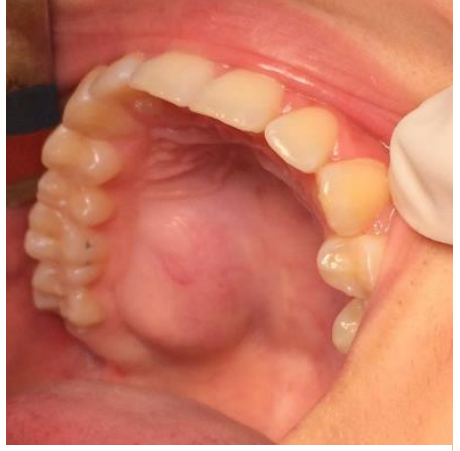

Figura 1 - Aspecto clínico inicial da lesão. nódulo extenso, de consistência flutuante, em palato duro, lado direito, na região dos dentes 14 ao 16, com recobrimento de mucosa integra e de coloração semelhante a mucosa adjacente e ausência de dor à palpação (Figura 1). O diagnóstico clínico foi de AP ou lesão cística em palato duro. A equipe solicitou

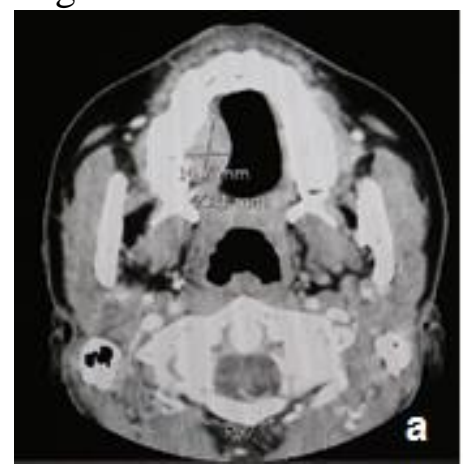

Figura 2 - TC de crânio pré-operatória evidenciando a lesão nodular em palato duro. a. Corte sagital. b. Corte coronal.

tomografia computadorizada (TC) da face, para avaliação e determinação de conduta. $\mathrm{O}$ laudo da TC indicou: lesão expansiva com impregnação heterogênea pelo meio de contraste medindo aproximadamente $1,4 \times 1,1$ $\mathrm{x} 2,3 \mathrm{~cm}$ em tecidos moles do palato duro à direita. A lesão causa remodelamento ósseo do palato em sua face oral. Não há sinal de extensão intracraniana ou neural da lesão. Não se identificam linfonodomegalias (Figura 2). Diante disso, foram solicitados exames pré-operatórios (hemograma completo, provas de coagulação, glicose) para posterior planejamento de biópsia incisional.

Os exames pré-operatórios mostraram valores dentro da normalidade e a paciente foi submetida à biópsia incisional, sob anestesia local, no Centro Cirúrgico Ambulatorial (CCA) (Figura 3).

O laudo do exame anatomopatológico indicou: adenoma pleomórfico de glândula salivar mucosa, em submucosa;

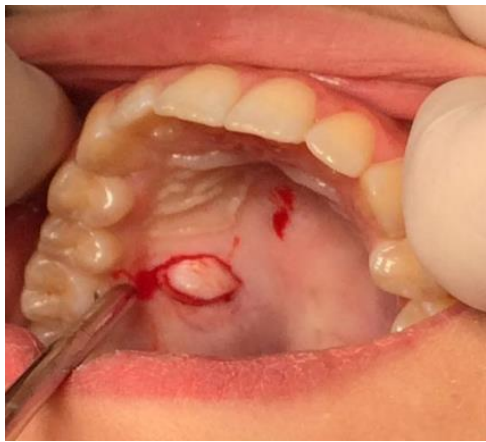

Figura 1 - Imagem transoperatória da biópsia incisional. 
presença de ceratose no epitélio superficial; ausência de neoplasia na amostra (Figura 4).
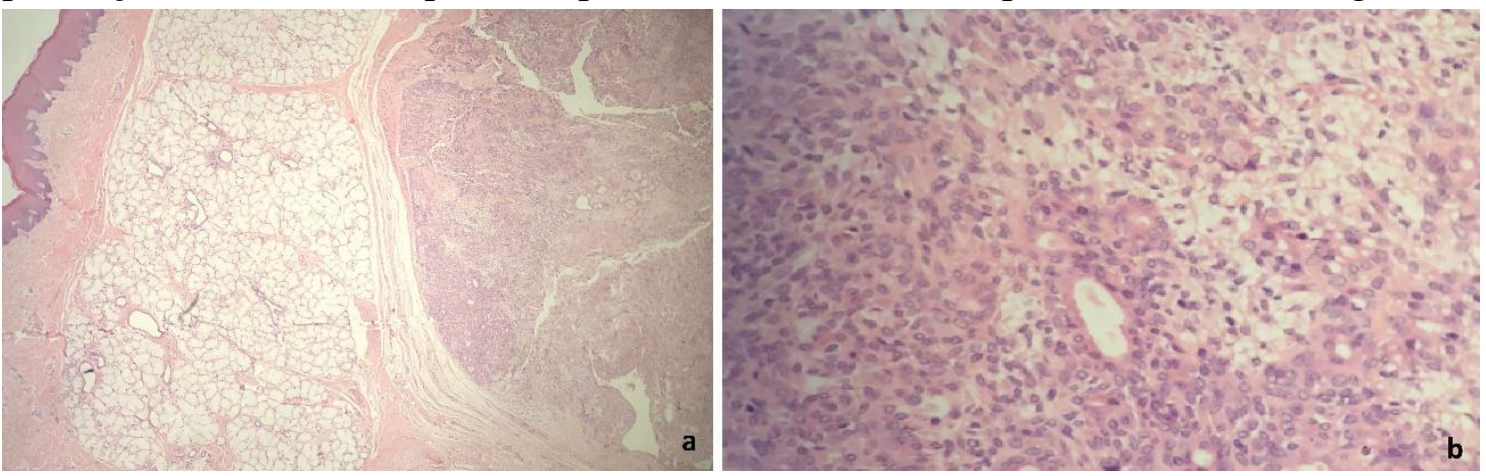

Figura 2 - Imagens do exame anatomopatológico. a. Adenoma pleomórfico, visão em menor aumento. $b$. Visão em maior aumento.

A exérese completa da lesão foi planejada em bloco cirúrgico sob anestesia geral. Realizou-se ampla ressecção cirúrgica até o tecido ósseo com margem de segurança de $1 \mathrm{~mm}$ em todo perímetro da lesão, confirmado por exame histopatológico de congelação transoperatório (Figura 5).

A ferida cirúrgica foi preenchida com cimento cirúrgico e instalou-se um aparelho ortodôntico em acrílico cobrindo a região para proteção durante alimentação e fala. Orientou-se a paciente a realizar bochechos com
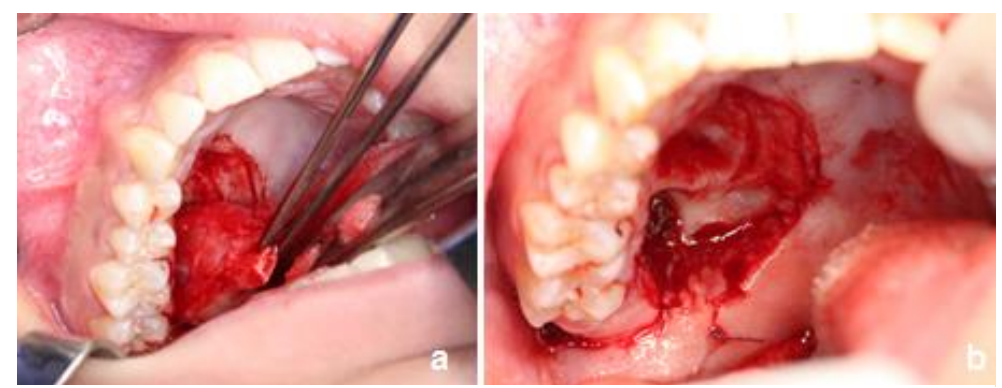
clorexidina $0,12 \%$ para higienização do local e prescreveu-se amoxicilina 500mg 8/8h por 7 dias e paracetamol $1 \mathrm{~g}$ de $6 / 6 \mathrm{~h}$ em caso de dor. Em 7 dias, foi realizada remoção total das suturas e troca dos bochechos de clorexidina por bochechos com água oxigenada 10 volumes diluída em proporção 1:3 a serem realizados 5 vezes ao dia. Com 20 dias de pós-operatório, foi possível observar a epitelização da ferida operatória. Com 60 dias de pós-operatório a ferida estava epitelizada, porém, uma depressão óssea ainda era observada na área. Foram
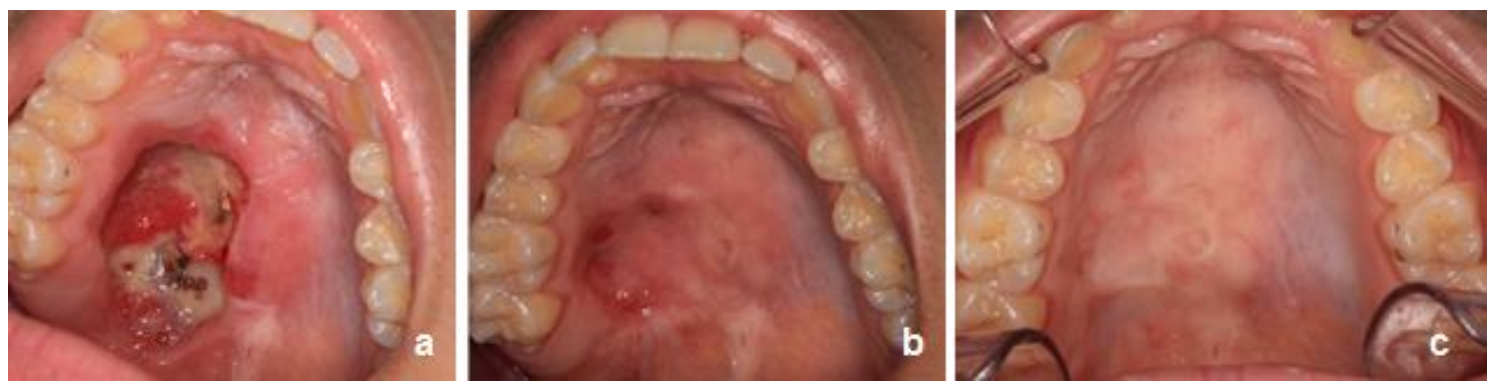

Figura 6 - Imagens do pós-operatório. A ferida cirúrgica com 16 dias de pós-operatório. b. Ferida cirúrgica com 60 dias de pós-operatório. c. Ferida cirúrgica com 1 ano de pós-operatório. 
realizados controles pós-operatórios periódicos, e com 18 meses, o exame clínico evidenciava reepitelização total dos tecidos moles e a TC de ossos da face não revelava recidiva da lesão, apresentando leve remodelação óssea (Figura 6). Durante todas as avaliações pós-operatórias em ambulatório a paciente não relatou dor ou desconforto.

\section{Discussão}

Diversos estudos epidemiológicos, realizados no Brasil e no mundo, indicam que os TGS são 75-80\% de natureza benigna. Destes apenas 5-25\% localizam-se em glândula salivar menor. A idade média de ocorrência dos TGS é 46 anos e observa-se uma forte predileção pelo sexo feminino. O AP é o TGS mais comum, ocorrendo aproximadamente em $80 \%$ dos casos diagnosticados $7,8,9,10,11$.

O AP é uma neoplasia de diagnóstico clínico, com confirmação a partir de exame anatomopatológico ${ }^{2}$. Embora seja uma lesão de crescimento normalmente lento, quando o tratamento não é realizado de maneira precoce, ele pode atingir grandes dimensões, o que leva a um aumento da morbidade do procedimento cirúrgico e pode levar a maiores taxas de recidiva e/ou transformação maligna ${ }^{2,4,5}$.

Para uma melhor acurácia clínica do diagnóstico, pode-se utilizar outros meios complementares de diagnóstico, como os exames de imagem e a citologia por punção aspirativa por agulha fina $(\mathrm{PAAF})^{12,13}$. O exame por imagem tem demonstrado papel de destaque no estabelecimento da origem, localização e limites da lesão. Por apresentar uma janela melhor para tecidos moles, há um consenso que as imagens por ressonância magnética (RNM) são melhores para o diagnóstico de TGS. Porém, algumas observações devem ser feitas: quando calcificações e áreas de necrose estão presentes no interior da massa tumoral, a TC revela-se superior, além de que, muitas vezes, a imagem por TC encontra-se mais facilmente disponível, o que justificaria sua indicação ${ }^{14}$.

Nos casos de AP no palato, após excisão cirúrgica, a ferida operatória apresentase cruenta e permanece exposta ao meio bucal. Visto que a boca desempenha funções essenciais como fala, mastigação e deglutição, os defeitos físicos deixados pelos procedimentos cirúrgicos podem afetar funcional e esteticamente o sistema estomatognático, afetando também a qualidade de vida dos pacientes ${ }^{15}$. Há um relato frequente que as áreas ósseas deixadas cicatrizar por segunda intenção provocam dores fortes nesses indivíduos ${ }^{16}$. Além da dor e do desconforto, outras complicações pósoperatórias podem ocorrer como hemorragia, necrose óssea, fístula oro-nasal e infecções ${ }^{15,16,17}$. Um adequado manejo cirúrgico pode evitar hemorragias e necrose tecidual. Um adequado controle pós-operatório, boa higienização do local operado e a proteção da região cruenta, diminuem o risco de infecções e favorecem a cicatrização da ferida $^{17}$.

Com o objetivo de tentar melhorar a qualidade de vida, diminuir a sensibilidade dolorosa dos pacientes durante o período de cicatrização e epitelização das feridas cirúrgicas, próteses obturadoras ou aparelhos acrílicos podem ser confeccionados para proteger a região cruenta ${ }^{18,19}$. A prótese obturadora melhora de maneira geral o funcionamento oral de pacientes com defeitos maxilares, oferecendo maior conforto 
durante as funções ${ }^{1819,20,21}$ e melhorando significativamente as habilidades de mastigação e deglutição ${ }^{20,21}$.

Há um consenso na literatura de que o tratamento mais adequado para AP seja sua excisão cirúrgica ${ }^{2,16,22}$. Entretanto, não há explicitado se este procedimento deva ocorrer sob anestesia local ou geral ${ }^{22}$. Para isso, deve-se levar em consideração alguns fatores como (1) tamanho e localização da lesão: quanto maior e mais próxima do palato mole, pode desencadear um reflexo de ânsia nos pacientes durante a manipulação cirúrgica; (2) tempo necessário para o procedimento cirúrgico: pode exigir maior permanência do paciente com a boca aberta e também um maior tempo de cirurgia pode levar a chances maiores de complicações pós-operatórias, como infecção; e (3) o risco de sangramento excessivo devido à proximidade com estruturas nobres, como a artéria palatina descendente, artéria palatina maior e artéria palatina menor ${ }^{23}$. Portanto a conduta terapêutica escolhida deve levar em consideração as particularidades de cada caso ${ }^{16,22,24}$.

\section{Conclusão}

O AP é uma lesão que merece destaque dentre os TGS devido a sua alta prevalência e chance de malignização/recidivas. Além disso, quando não tratados adequadamente podem se tornar grandes massas tumorais e trazer enorme morbidade aos pacientes. Salientamos a importância de um diagnóstico preciso e um tratamento efetivo com a maior brevidade. Quando possível, as próteses obturadoras/aparelhos intraorais acrílicos podem e devem ser utilizados de forma associada aos tratamentos cirúrgicos de tumores, restabelecendo função e trazendo conforto pós-operatório.

\section{REFERÊNCIAS}

1. Sailer HF, Pajarola GF. Doenças das glândulas salivares. In: Atlas colorido de odontologia: Cirurgia Bucal. Porto Alegre: Artmed; 2000. p.241-6.

2. Neville B. Patologia oral e maxilofacial. 3. ed. Rio de Janeiro: Elsevier; 2009.

3. Sunil S, Gopakumar D. Adenoma pleomorfico. Reporte de caso y revision de la literatura. Int. J. Odontostomat. 2013;7(2):171-4.

4. Tiago RSL, Castro GA, Ricardo LAC, Bühler RB, Fava AS. Adenoma pleomórfico de parótida: aspectos clínicos, diagnósticos e terapêuticos. Rev Bras Otorrinolaringol. 2003;69(4):485-9.

5. Bordoy-Soto MA, Véles-Gimón HJ, Hernández MF, Xavier-Briceño, W. Giant pleomorphic adenoma of the palate. Case report and literature review. Revista Odontológica Mexicana 2016;20(4):252-7.

6. Sarmento DJ, Morais ML, Costa AL, Silveira ÉJ. Neoplasias intraorais de glândula salivar menor: estudo clínico-patológico. Einstein 2016;14(4):508-12.

7. Vargas PA, Gerhard R, Araújo Filho VJF, Castro IV. Salivary gland tumors in a Brazilian population: a retrospective study of 124 cases. Rev. Hosp. Clín. Fac. Med. S. Paulo 2002;57(6):271-276. 
8. Vasconcelos AC, Nör F, Meurer L, Salvadori G, Souza LB, Vargas PA et al. Clinicopathological analysis of salivary gland tumors over a 15-year period. Braz Oral Res [online]. 2016;30(e2):1-7.

9. Lima SS, Soares AF, Amorim RFB, Freitas RA. Perfil epidemiológico das neoplasias de glândulas salivares: análise de 245 casos. Rev Bras Otorrinolaringol. 2005;71(3):335-40.

10. Gao M, Hao Y, Huang MX, Ma DQ, Chen Y, Luo HY et al. Salivary gland tumours in a northern Chinese population: a 50- year retrospective study of 7190 cases. Int. J. Oral Maxillofac. Surg. 2017; 46: 343- 49.

11. Oliveira FA, Duarte ECB, Taveira CT, Máximo AA, Aquino EC, Alencar RC et al. Salivary Gland Tumor: A Review of 599 Cases in a Brazilian Population. Head and Neck Pathol. 2009;3:271-5.

12. Gonçalves AJ, Menezes MB, Kavabata NK, Bertelli AAT, Souza RAS, Joelsons D. Punção aspirativa nos tumores das glândulas salivares: especificidade e sensibilidade. Rev Assoc Med Bras. 2007;53(3):267-71.

13. Gudmundsson JK, Ajan A, Abtahi J. The acuracy of fine-needle aspiration citology for diagnosis of parotid gland masses: a clinicopathological study of 114 patients. J Appl Oral Sci. 2016;24(6):561-7.

14. Ribeiro-Rotta RF, Cruz ML, Paiva RR, Mendonça EF, Spini TH, Mendonça AR. O papel da ressonância magnética no diagnóstico do adenoma pleomórfico: revisão da literatura e relato de casos. Rev Bras Otorrinolaringol. 2003;69(5):699707.

15. Hirota SK, Penha SS, Lehn CN, Sugaya NN, Migliare DA. Quality of life in patients submitted to surgical treatment for minor salivary gland neoplasms. Braz Oral Res. 2007;21(4):375-9.

16. Oliveira LJ, Castro HHO, Leão PLR, Leal RM, Horta MCR, Souza PEA. Tratamento de adenoma pleomórfico em palato: relato de 2 casos e revisão de literatura. Rev Port Estomatol Med Dent Cir Maxilofac. 2016;57(1):55-61.

17. Fernandes JBVD, Nunes TP, Matayoshi S, Moura EM. Enxerto de mucosa do palato duro: complicações na área doadora - Relato de casos. Arq Bras Oftalmol 2003;66:884-6.

18. Chen C, Ren W, Gao L, Cheng Z, Zhang L, Li S et al. Function of obturator prosthesis after maxillectomy and prosthetic obturator rehabilitation. Braz J Otorhinolaryngol. 2016;82(2):177-83.

19. Santos DM, Nagay BE, Bitencourt SB, Silva EVF, Goiato MC. Reabilitação com prótese obturadora após maxilectomia parcial: relato de caso. Revista Odontológica de Araçatuba 2016;37(2):52-66.

20. Kreeft M, Krap M, Wismeijer D, Speksnijder CM, Smeele LE, Bosch SD et al. Oral function after maxillectomy and reconstruction with an obturator. Int. J. Oral Maxillofac. Surg. 2012; 41:1387-92. 
21. Vero N, Mishra N, Singh BP, Singh K, Jurel SK, Kumar V. Assessment of swallowing and masticatory performance in obturator wearers: a clinical study. $\mathrm{J}$ Adv Prosthodont 2015;7:8-14.

22. Santos HKA, Damasceno RVM, Cardoso JÁ, Cancio AV, Farias JG. Relatos de tratamentos distintos para o adenoma pleomórfico. Rev. Cir. Traumatol. BucoMaxilo-Fac. 2016;16(3):53-8.

23. Shahbazi A, Grimm A, Feigl G, Gerber G, Székely AD, Molnár B et al. Analysis of blood supply in the hard palate and maxillary tuberosity — clinical implications for flap design and soft tissue graft harvesting (a human cadaver study). Clin Oral Invest. $2018 \mathrm{Jul}$ 1. doi: 10.1007/s00784-018-2538-3. [Epub ahead of print].

24. Shigeishi H, Ohta K, Takechi M. Risk factors for postoperative complications following oral surgery. J Appl Oral Sci. 2015;23(4):419-23. 